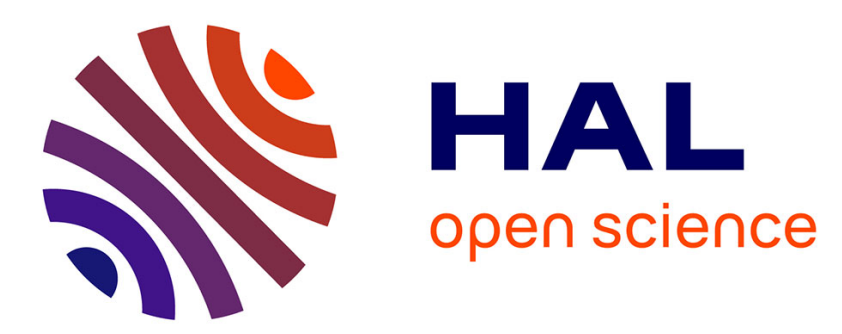

\title{
Source Localization by Gradient Estimation Based on Poisson Integral
}

\author{
Ruggero Fabbiano, Carlos Canudas de Wit, Federica Garin
}

\section{To cite this version:}

Ruggero Fabbiano, Carlos Canudas de Wit, Federica Garin. Source Localization by Gradient Estimation Based on Poisson Integral. Automatica, 2014, 50 (6), pp.1715-1724. 10.1016/j.automatica.2014.04.029 . hal-00999417

\section{HAL Id: hal-00999417 https://inria.hal.science/hal-00999417}

Submitted on 3 Jun 2014

HAL is a multi-disciplinary open access archive for the deposit and dissemination of scientific research documents, whether they are published or not. The documents may come from teaching and research institutions in France or abroad, or from public or private research centers.
L'archive ouverte pluridisciplinaire HAL, est destinée au dépôt et à la diffusion de documents scientifiques de niveau recherche, publiés ou non, émanant des établissements d'enseignement et de recherche français ou étrangers, des laboratoires publics ou privés. 
Brief paper

\title{
Source localization by gradient estimation based on Poisson integral ${ }^{\star x}$
}

\author{
Ruggero Fabbiano ${ }^{\mathrm{a}, 1}$, Carlos Canudas de Wit ${ }^{\mathrm{b}}$, Federica Garin ${ }^{\mathrm{a}}$ \\ a Inria, NeCS team, 655 avenue de l'Europe, 38334 Montbonnot Saint Martin, France \\ b CNRS, Control System department, GIPSA-lab, NeCS team, 11 rue des Mathématiques, 38400 Saint Martin d'Hères, France
}

\section{A R T I C L E I N F O}

\section{Article history:}

Received 6 May 2013

Received in revised form

3 December 2013

Accepted 26 March 2014

Available online $\mathrm{xxxx}$

\section{Keywords:}

Source seeking

Sensor network

Gradient search

Poisson integral

\begin{abstract}
A B S T R A C T
We consider the problem of localizing the source of a diffusion process. The source is supposed to be isotropic, and several sensors, equipped on a vehicle moving without position information, provide pointwise measures of the quantity being emitted. The solution we propose is based on computing the gradient - and higher-order derivatives such as the Hessian - from Poisson integrals: in opposition to other solutions previously proposed, this computation does neither require specific knowledge of the solution of the diffusion process, nor the use of probing signals, but only exploits properties of the PDE describing the diffusion process. The theoretical results are illustrated by simulations.
\end{abstract}

(C) 2014 Elsevier Ltd. All rights reserved.

\section{Introduction}

The problem of source localization consists in finding, by one or several mobile or fixed sensors arrays, possibly cooperating with each other, the point or the spatial region from which a quantity of interest is being emitted. Source-seeking agents can be fixed sensors, that collect and exchange some information about the signal field and try to identify the position of the source (or the smallest region in which it is included), or moving devices equipped with one or more sensors, that physically reach the source in an individual or cooperative way.

This research area is attracting rapidly increasing interest, in particular in applications where the agents have limited or no position information; for instance, source localization is relevant to many applications of vapor emitting sources (Porat \& Nehorai, 1996), such as explosive detection, drug detection, sensing leaking or hazardous chemicals, pollution sensing and environmental studies. Sound source localization (Zhang, Florêncio, Ba, \& Zhang, 2008) is pertinent for intelligent conference call systems that identify the speakers to improve sound and video quality. Other

\footnotetext{
The material in this paper was partially presented at the 3rd IFAC Workshop on Distributed Estimation and Control in Networked Systems (NecSys'2), September 14-15, 2012, Santa Barbara, California, USA. This paper was recommended for publication in revised form by Associate Editor Xiaobo Tan under the direction of Editor Miroslav Krstic.

E-mail addresses: ruggero.fabbiano@inria.fr (R. Fabbiano), carlos.canudas-de-wit@gipsa-lab.fr (C.C. de Wit), federica.garin@inria.fr (F. Garin).

1 Tel.: +334 7661 5532; fax: +33 476615455 .
}

applications also include heat source localization, vent sources in underwater field, and medical applications to explore internal brain activity by using surface sensors.

\subsection{Overview of source-seeking}

A variety of methods exists in the literature to treat the problem of source localization and related issues. Many techniques deal with formulations associated with isotropic diffusion processes, and several identification methods have been devised to estimate the source position (Matthes, Gröll, \& Keller, 2004; Porat \& Nehorai, 1996); more fundamental problems, such as source identifiability and optimal sensor placement, are discussed in depth in Khapalov (2010). This approach, that can be viewed as an inverse problem formulation for partial differential equations, has the drawbacks of a heavy computation, and the requirement of the explicit knowledge of the closed-form solution of the PDE describing the diffusion process.

A different line of research consists in reconstructing an approximation of the gradient field of the measured quantity, and moving towards the source along the gradient direction; this can be done either directly, via a method developed for the particular problem at hand, or implicitly, by estimating the gradient via different techniques. The first contribution making use of an explicit ad hoc gradient computation can be found in Burian, Yoerger, Bradley, and Singh (1996), where the agent obtains different measurements of a hydrothermal plume and performs a least-square gradient estimation; in Baronov and Baillieul (2008) unicycle vehicles are driven towards a source by a control law 
related to the geometry of the diffusion process. The gradient is estimated in an implicit way in Wu, Couzin, and Zhang (2012), where the authors take inspiration from fish swarms to design an algorithm adapted also for seeking the source of a turbulent flow (Wu, Chang, \& Zhang, 2013).

In contrast to the previously mentioned methods, the so-called "extremum seeking" methodology is not based on any particular structure or knowledge of the diffusion solution; the method only applies for moving sensors, as it relies on the idea of collecting rich enough information to approximate the gradient through the use of a periodic probing signal or an oscillatory motion. Extremum-seeking has been applied in a wide range of engineering applications; adaptations of this idea to the problem of source localization are presented in Ghods (2011) and references therein, where the control of the nonholonomic unicycle is performed first on the forward velocity, then on angular one, while in the last case the authors combine the two strategies in an elegant way. Extremum-seeking has been used also in some contributions on 3D source localization (Cochran, Ghods, \& Krstić, 2008; Cochran, Siranosian, Ghods, \& Krstić, 2009).

Another technique worth mentioning is given by stochastic source-localization. Stochastic methods are based on a function that describes the probability rate of a change of direction, and often mimic biological behaviors observed for example on fish swarms or in bacteria movements. A contribution in this direction is the Optimotaxis (Mesquita, Hespanha, \& AAström, 2008), where the vehicles move with a random motion similar to Escherichia coli's "run and tumble"; this method can be also used with nonquadratic-like signal profiles, including the ones with multiple maxima. Another contribution can be found in Menon and Ghose (2012), where the authors localize a source of polluting substance and also track the boundary of the contaminated region.

All the methods discussed above have been also used to develop a distributed approach to the source-localization problem. One of the first in this direction is (Moreau, Bachmayer, \& Leonard, 2003), where it is assumed that each vehicle, modeled with simple integrator dynamics, can measure the full gradient, and the authors develop a twofold algorithm with a gradientdescent term and inter-vehicle forcing terms. Extremum-seeking is applied in a collaborative manner in Ghods and Krstić (2010), in a 1-dimensional framework. Two distributed stochastic source localizations are in Sahyoun, Djouadi, and Qi (2010), where a group of chemical sensors takes measures of a plume concentration values to estimate the source position via a stochastic approximation technique, and in Rabbat and Nowak (2004), in which the authors use the sensor measurements to estimate the model parameters of the concentration plume. A collaborative control law to steer a fleet of AUVs (autonomous underwater vehicles) to the source of a signal distribution using only direct signal measurements by a circular formation of agents is presented in Briñón Arranz, Seuret, and Canudas de Wit (2011) and Moore and Canudas de Wit (2010); these ideas are formalized and extended in Canudas de Wit, Garin, Fabbiano, Rouchon, and Rousseau (2012). Some recent works using a distributed approach deal in particular with acoustic source localization; an example is (Yong, Qing-Hao, Yuxiu, \& Ming, 2012), in which the authors solve the so-called "energy-based source localization" problem, i.e., detecting the presence of a source emitting an acoustic signal that attenuates in space by a field of sensors able to measure the signal's energy, by proposing a new optimization method called "projection onto the nearest local minimum".

\subsection{Main paper contribution}

We present here a new method, suitable for a single-vehicle n-dimensional source localization, based on a direct gradient computation. This technique does not require specific knowledge of the solution of the diffusion process, but only exploits properties of the PDE that generates the diffusion process, and can compute the gradient direction from the pointwise concentration samples collected by multiple sensors arranged on a spherical surface, with a small computation load; it does not make use of a probing signal either. We note also that the vehicle does not need any position information, since the heading references can be computed with respect to the vehicle's orientation in its local frame.

The gradient computation, necessary to perform the source search, is based on the Poisson integral formula; this approach allows also for higher-order derivatives computation (e.g., the Hessian), which can be useful to implement different control laws. Moreover, this is intrinsically high-frequency filtering, since derivatives are computed using integrals, and it makes the method less sensitive to measurement noise.

The approach is based on the assumption, justified for isotropic diffusive sources in steady-state, that the diffusion process is described by the Laplacian PDE. The paper formalizes and extends previous ideas from Briñón Arranz et al. (2011) and Moore and Canudas de Wit (2010), where the gradient has been approximated by the sum of pointwise measurements around a circle weighted by the position vector of each sampler with respect to its center of rotation.

\section{Problem formulation}

Before starting we fix here some notation we will use throughout the paper. A point in an $n$-dimensional space is represented by the vector $\boldsymbol{x}=\left[\begin{array}{llll}x_{1} & x_{2} & \cdots & x_{n}\end{array}\right]^{\mathrm{T}} ; \nabla f$ is the gradient of the function $f$, and $\nabla^{2}=\sum_{i=1}^{n} \partial^{2} / \partial x_{i}^{2}$ is the Laplacian operator. For an open set $\Omega, \partial \Omega$ denotes its border, and $\bar{\Omega}=\Omega \cup \partial \Omega$ its closure. $B_{r}(\boldsymbol{c})$ indicates the ball of radius $r$ centered in $\boldsymbol{c}$; the area of the surface of $B_{r}(\boldsymbol{c}) \subset \mathbb{R}^{n}$ is given by $\omega_{n} r^{n-1}, \omega_{n}$ being the area of the corresponding unit sphere, and $d S_{B_{r}(\boldsymbol{c})}$ denotes the infinitesimal element of surface of $B_{r}(\boldsymbol{c})$. Integrals of vectoror matrix-valued quantities are intended as entry-wise integrals. Finally, [a] denotes the rounding of $a$, i.e., the integer closest to $a$.

We consider steady-state behaviors of homogeneous diffusion processes caused by an isotropic source emitting at a constant rate. Such a process is governed by the well-known diffusion equation

$$
\frac{\partial f(\boldsymbol{x}, t)}{\partial t}-k \nabla^{2} f(\boldsymbol{x}, t)=0, \quad \forall \boldsymbol{x} \in \Omega, t \geq 0,
$$

where $f$ is the concentration variable, $k$ is a diffusion coefficient, and $\Omega \subset \mathbb{R}^{n}$ (see Folland, 1995). In particular, as depicted in Fig. 1(a), we consider the region of interest $\tilde{\Omega} \subset \mathbb{R}^{n}$ as a connected bounded set $\tilde{\Omega}=\Omega \cup \Omega_{\mathrm{s}}$, where $\Omega_{\mathrm{s}}$ is the connected bounded set that identifies the source, and therefore we have that $\partial \Omega=\partial \tilde{\Omega} \cup$ $\partial \Omega_{\mathrm{s}}$. The values of $f$ on the inner boundary $\partial \Omega_{\mathrm{s}}$ are imposed by the source, so we can assume that values of $f$ on $\partial \Omega_{\mathrm{s}}$ are higher than the ones on $\partial \tilde{\Omega}$. As we will see below, we have that $\max f(\boldsymbol{x}) \in \partial \Omega$, that means, for our previous consideration, that it lies on $\partial \Omega_{s}$; our source localization problem is then mathematically equivalent to the problem of finding the maximum of $f$.

Once the steady-state has been reached, supposing that the source is still emitting at a constant rate (this happens in many cases of practical interest, e.g., in a heating process, or in the dispersion of a chemical substance), or that possible source variations are slow in the time-scale of interest, the diffusion equation (1) reduces to the Laplace equation

$\nabla^{2} f(\boldsymbol{x})=0, \quad \forall \boldsymbol{x} \in \Omega$,

whose solutions are called "harmonic". These functions have many properties; among them, they satisfy the maximum principle, which 


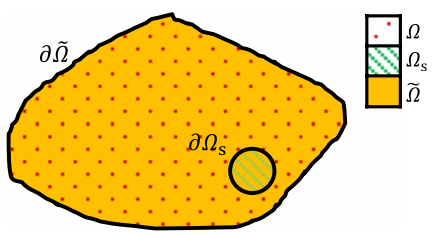

(a) Sets as defined in Section 2: $\partial \Omega=\partial \tilde{\Omega} \cup \partial \Omega_{\mathrm{s}}$.

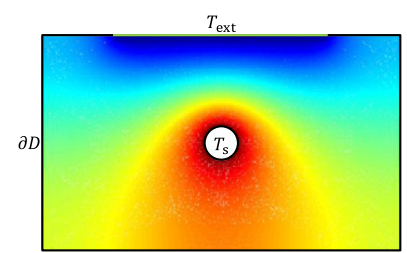

(b) Solution of the problem (4).

Fig. 1. Representations of the domain for the problem formulation.

ensures that the distribution has a global maximum on $\partial \Omega$ and which we recall below ${ }^{2}$ :

Proposition 1 (Maximum Principle). Let $\Omega \subset \mathbb{R}^{n}$ be a bounded open set, and let $f: \bar{\Omega} \rightarrow \mathbb{R}$ be continuous in $\bar{\Omega}$ and harmonic in $\Omega$. Then, the maximum value of $f$ on $\bar{\Omega}$ is achieved on $\partial \Omega$.

Proposition 2 (Local Maximum Principle). Let $\Omega \subseteq \mathbb{R}^{n}$ be a connected open set, and let $f: \Omega \rightarrow \mathbb{R}$ be harmonic on $\Omega$. If $f$ has a local maximum in $\Omega$, then $f$ is constant.

Example 1. As an example of a harmonic function we can consider the function

$f(\boldsymbol{x})=\frac{A}{\left\|\boldsymbol{x}-\boldsymbol{x}_{\mathrm{s}}\right\|_{2}}$,

with $\boldsymbol{x} \in \mathbb{R}^{3}$. This function is represented by spherical level sets centered at the given point $\boldsymbol{x}_{\mathrm{s}}$. It is easy to verify that $\nabla^{2} f(\boldsymbol{x})=$ $0 \forall \boldsymbol{x} \neq \boldsymbol{x}_{\mathrm{s}}$, showing that the function is harmonic on $\mathbb{R}^{3} \backslash\left\{\boldsymbol{x}_{\mathrm{s}}\right\}$. Throughout the paper, unless otherwise stated, we will consider the domain of interest as $\Omega=\mathbb{R}^{3} \backslash \Omega_{\mathrm{s}}$, where $\Omega_{\mathrm{s}}=B_{\varepsilon}\left(\boldsymbol{x}_{\mathrm{s}}\right)$.

Example 2. In some cases, a harmonic function cannot be written in closed-form, but it can be expressed as the solution of a PDE problem. As an example, let us consider the rectangular bounded domain $\bar{D}=[0, a] \times[0, b], b<a$, and the circle $B_{r}(a / 2, b / 2), r \ll$ $b / 2$. The solution of the mixed Dirichlet-Neumann problem

$$
\begin{cases}\nabla^{2} f(\boldsymbol{x})=0, & \text { in } D \backslash B_{r}\left(\frac{a}{2}, \frac{b}{2}\right) \quad \text { (a) } \\ \nabla f(\boldsymbol{x}) \cdot \hat{\boldsymbol{n}}=0, & \text { on } \partial D \backslash[c, a-c] \times b \\ f(\boldsymbol{x})=T_{\mathrm{ext}}, & \text { on }[c, a-c] \times b \quad \text { (c) } \\ f(\boldsymbol{x})=T_{\mathrm{s}}, & \text { on } \bar{B}_{r}\left(\frac{a}{2}, \frac{b}{2}\right) \quad \text { (d), }\end{cases}
$$

with $c<a$, and $\hat{\boldsymbol{n}}$ the outward normal to the domain's borders, is a function $f$ harmonic in $\bar{D}$. It cannot be written in closed form, but can be obtained by a finite-elements method as a numerical solution, and its profile is represented in Fig. 1(b).

Thanks to the property of harmonicity of the function describing our source emission, we can develop a way to approximate it, as well as its derivatives, with a finite sum of pointwise measurements, as we are going to show in the next section. Moreover, the maximum principles ensure we have no local maxima inside $\Omega$, so it is possible to use a gradient-search technique to localize the source.

\section{Derivative computation}

\subsection{Poisson integral for the ball}

We will show how it is possible to compute values of the function $f$ solution of the Laplace equation (2), and thence of

\footnotetext{
2 The two following statements, as well as Theorem 1, are taken from Axler, Bourdon, and Ramey (2001) and Folland (1995), to which we refer for a more detailed study of the properties of harmonic functions.
}

its derivatives of any order (in particular, the gradient and the Hessian), at any point in the domain $\Omega$, only by measuring its values on a surface enclosing the point.

For some regular domains this value can be expressed by an integral, called Poisson integral, of the product between the values of the function on the border surface and a function called Poisson kernel. For example, given the unit sphere, the Poisson kernel and the Poisson integral are defined as follows (Axler et al., 2001).

Definition 1 (Poisson Kernel for the Unit Ball). Given a generic point $\boldsymbol{x} \in B_{1}(0)$ inside the unit ball of dimension $n$, and $\overline{\boldsymbol{x}} \in \partial B_{1}(0)$ being a point on the outer boundary, i.e., on the unit ball surface, we call Poisson kernel for the unit ball the function

$P_{B_{1}(0)}(\boldsymbol{x}, \overline{\boldsymbol{x}})=\frac{1-\|\boldsymbol{x}\|^{2}}{\|\boldsymbol{x}-\overline{\boldsymbol{x}}\|^{n}}$.

Theorem 1 (Poisson Integral for the Unit Ball). Let $f: \bar{B}_{1}(0) \rightarrow \mathbb{R}$ be continuous on the closed unit sphere $\bar{B}_{1}(0)$, harmonic on $B_{1}(0)$. Then $f$ is, on $B_{1}(0)$, the Poisson integral of its restriction on $\partial B_{1}(0)$, namely:

$f(\boldsymbol{x})=\frac{1}{\omega_{n}} \int_{\partial B_{1}(0)} P_{B_{1}(0)}(\boldsymbol{x}, \overline{\boldsymbol{x}}) f(\overline{\boldsymbol{x}}) \mathrm{d} S_{B_{1}(0)}(\overline{\boldsymbol{x}})$,

for all $\boldsymbol{x} \in B_{1}(0)$.

Then, by mapping the point $\boldsymbol{x}$ to the point $\frac{\boldsymbol{x}-\boldsymbol{c}}{r}$, it is possible to obtain the analogous formula for the generic ball $B_{r}(\boldsymbol{c})$.

Theorem 2 (Poisson Integral for the Generic Ball). Let $f: \bar{B}_{r}(\boldsymbol{c}) \rightarrow \mathbb{R}$ be continuous on $\bar{B}_{r}(\boldsymbol{c})$, harmonic on $B_{r}(\boldsymbol{c})$. Then:

$f(\boldsymbol{x})=\frac{1}{\omega_{n} r} \int_{\partial B_{r}(\boldsymbol{c})} P_{B_{r}(\boldsymbol{c})}(\boldsymbol{x}, \overline{\boldsymbol{x}}) f(\overline{\boldsymbol{x}}) \mathrm{d} S_{B_{r}(\boldsymbol{c})}(\overline{\boldsymbol{x}})$,

where $\boldsymbol{x} \in B_{r}(\boldsymbol{c})$, and $P_{B_{r}(\boldsymbol{c})}$ is the Poisson kernel for the generic ball, defined as

$P_{B_{r}(\boldsymbol{c})}(\boldsymbol{x}, \overline{\boldsymbol{x}}):=\frac{r^{2}-\|\boldsymbol{x}-\boldsymbol{c}\|^{2}}{\|\boldsymbol{x}-\overline{\boldsymbol{x}}\|^{n}}$.

Hence, if we consider a function $f$ harmonic on an open set $\Omega \subseteq$ $\mathbb{R}^{n}$, we can compute the value of $f$ at any point $\boldsymbol{x} \in \Omega$ by applying (5) to any ball such that its closure is contained in $\Omega$. In particular, when evaluated in the center of the ball, the Poisson kernel in (6) reduces to

$P_{B_{r}(\boldsymbol{c})}(\boldsymbol{c}, \overline{\boldsymbol{x}})=\frac{r^{2}}{r^{n}}=\frac{1}{r^{n-2}}$,

and the expression for $f(\boldsymbol{x})$ from (5) becomes much simpler:

$f(\boldsymbol{c})=\frac{1}{\omega_{n} r^{n-1}} \int_{\partial B_{r}(\boldsymbol{c})} f(\overline{\boldsymbol{x}}) \mathrm{d} S_{B_{r}(\boldsymbol{c})}(\overline{\boldsymbol{x}})$.

\subsection{Gradient computation}

The Poisson integral given in (5) also allows for the computation of derivatives of $f$ in the same way, as stated in the next proposition. 


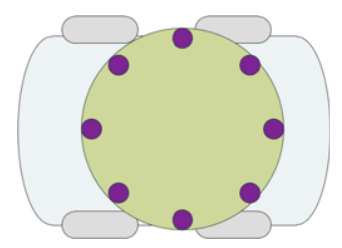

(a) Planar vehicle with a circular array of sensors.

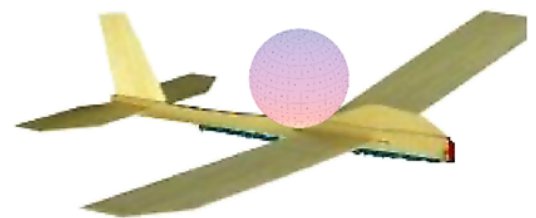

(b) Tridimensional vehicle e-quipped with the sensing device.

Fig. 2. Source-seeking vehicles suitable for $2 \mathrm{D}$ and 3D scenarios.

Proposition 3. Let $\Omega \subseteq \mathbb{R}^{n}$ be an open set, and $f: \Omega \rightarrow \mathbb{R}$ be harmonic on $\Omega$. For any $\bar{c} \in \Omega$, for any $r>0$ such that $\bar{B}_{r}(\mathbf{c}) \subseteq \Omega$, for any $\boldsymbol{x} \in B_{r}(\boldsymbol{c})$, and for any non-negative integers $m_{1}, \ldots, m_{n}$ such that $\sum_{i=1}^{n} m_{i}=m$,

$$
\frac{\partial^{m} f(\boldsymbol{x})}{\prod_{i=1}^{n} \partial x_{i}^{m_{i}}}=\frac{1}{\omega_{n} r} \int_{\partial B_{r}(\boldsymbol{c})} \frac{\partial^{m} P_{B_{r}(\boldsymbol{c})}(\boldsymbol{x}, \overline{\boldsymbol{x}})}{\prod_{i=1}^{n} \partial x_{i}^{m_{i}}} f(\overline{\boldsymbol{x}}) \mathrm{d} S_{B_{r}(\boldsymbol{c})}(\overline{\boldsymbol{x}}) .
$$

Proof. From the assumptions, we can apply Theorem 2. Then, it is possible to bring the derivation operator, which is with respect to $\boldsymbol{x}$, inside the integral, which is with respect to $\overline{\boldsymbol{x}}$.

Applying (8) it is easy to obtain the expression of the gradient $\nabla f(\boldsymbol{c})$ : the gradient of the Poisson kernel can be computed from (6) as

$\nabla P(\boldsymbol{c}, \overline{\boldsymbol{x}})=\left.\frac{\partial}{\partial \boldsymbol{x}} \frac{r^{2}-\|\boldsymbol{x}-\boldsymbol{c}\|^{2}}{\|\boldsymbol{x}-\overline{\boldsymbol{x}}\|^{n}}\right|_{\boldsymbol{x}=\boldsymbol{c}}=\frac{n}{r^{n}} r \hat{\boldsymbol{n}}=\frac{n}{r^{n-1}} \hat{\boldsymbol{n}}$,

where $\hat{\boldsymbol{n}}$ is the normal outward unit vector, $\hat{\boldsymbol{n}}=\frac{\overline{\boldsymbol{x}}-\boldsymbol{c}}{r}$. This gives us

$$
\begin{aligned}
\nabla f(\boldsymbol{c}) & =\frac{1}{\omega_{n} r} \int_{\partial B_{r}(\boldsymbol{c})} \nabla P(\boldsymbol{c}, \overline{\boldsymbol{x}}) f(\overline{\boldsymbol{x}}) \mathrm{d} S_{B_{r}(\boldsymbol{c})}(\overline{\boldsymbol{x}}) \\
& =\frac{n}{\omega_{n} r^{n}} \int_{\partial B_{r}(\boldsymbol{c})} \hat{\boldsymbol{n}} f(\overline{\boldsymbol{x}}) \mathrm{d} S_{B_{r}(\boldsymbol{c})}(\overline{\boldsymbol{x}}) .
\end{aligned}
$$

Similarly, we can compute the Hessian $\boldsymbol{H}_{f}(\boldsymbol{c})$; after computation of the Hessian of the Poisson kernel, we get

$$
\begin{aligned}
\boldsymbol{H}_{f}(\boldsymbol{c}) & =\frac{1}{\omega_{n} r} \int_{\partial B_{r}(\boldsymbol{c})} \boldsymbol{H}_{\mathrm{P}}(\boldsymbol{c}, \overline{\boldsymbol{x}}) f(\overline{\boldsymbol{x}}) \mathrm{d} S_{B_{r}(\boldsymbol{c})}(\overline{\boldsymbol{x}}) \\
& =\frac{n+2}{\omega_{n} r^{n+1}} \int_{\partial B_{r}(\boldsymbol{c})}\left(n \hat{\boldsymbol{n}} \hat{\boldsymbol{n}}^{\mathrm{T}}-\boldsymbol{I}\right) f(\overline{\boldsymbol{x}}) \mathrm{d} S_{B_{r}(\boldsymbol{c})}(\overline{\boldsymbol{x}}),
\end{aligned}
$$

with $I$ the identity matrix of size $n$.

Remark 1. Eq. (9a) becomes exact for every function $f \in \mathcal{C}^{1}(\boldsymbol{c})$ when the radius tends to zero. Indeed, as already shown in Briñón Arranz et al. (2011, Lemma 1) for the gradient approximation in two dimensions, we can write the first-order Taylor expansion

$f(\overline{\boldsymbol{x}})=f(\boldsymbol{c}+r \hat{\boldsymbol{n}})=f(\boldsymbol{c})+\nabla f(\boldsymbol{c}) \cdot r \hat{\boldsymbol{n}}+o(r)$

and, multiplying both sides by $\hat{\boldsymbol{n}}$ and integrating over the ball, we get

$$
\begin{aligned}
\int_{\partial B_{r}(\boldsymbol{c})} f(\overline{\boldsymbol{x}}) \hat{\boldsymbol{n}} \mathrm{d} S_{B_{r}(\boldsymbol{c})}(\overline{\boldsymbol{x}})= & f(\boldsymbol{c}) \int_{\partial B_{r}(\boldsymbol{c})} \hat{\boldsymbol{n}} \mathrm{d} S_{B_{r}(\boldsymbol{c})}(\overline{\boldsymbol{x}}) \\
& +r \int_{\partial B_{r}(\boldsymbol{c})}(\nabla f(\boldsymbol{c}) \cdot \hat{\boldsymbol{n}}) \hat{\boldsymbol{n}} \mathrm{d} S_{B_{r}(\boldsymbol{c})}(\overline{\boldsymbol{x}})+o\left(r^{n}\right) .
\end{aligned}
$$

The first integral on the right-hand side is equal to zero, while the second gives $\omega_{n} r^{n-1} n^{-1} \nabla f(\boldsymbol{c})$, leading us to (9a) up to an error $o\left(r^{n}\right)$. We present here a framework in which, under additional assumptions on $f$, and provided that the ball $B_{r}(\boldsymbol{c})$ is contained in $\Omega$, the formulas for the gradient and Hessian computation are exact for any radius.
Remark 2. The problem of finding the value of a harmonic function inside a domain, given its values on the border of the domain itself, is well-defined for every shape of the domain border. However, it is not possible to write the Poisson kernel in a closedform for domains other than the half-plane and the sphere.

In the next section we are going to develop a source-seeking strategy based on the formulas here introduced.

\section{Source-seeking strategy}

Steering the vehicle towards the source can be obtained by performing a gradient-ascent method based on the formulas derived previously, with the gradient (and possibly the Hessian) being estimated by collecting pointwise measurements of the quantity of interest. Hence, we need the vehicle to be equipped with a sensing device that can take measurements on a ball of suitable dimension, as shown in Fig. 2.

In the next subsection we briefly consider the bidimensional case, whose equations are more immediate and for which we refer to our previous work (Canudas de Wit et al., 2012). Then, we focus more deeply on the three-dimensional one.

\subsection{Dynamics and control law for planar source-seeking}

The central idea is that we can approximate the integrals in (7) and (9) by the Riemann sum of the $N$ values provided by the sensors arranged along the circle (see Fig. 2(a)). Using the polar coordinates $r \hat{\boldsymbol{n}}=r(\cos \theta, \sin \theta), \theta \in[0,2 \pi)$, with $\omega_{2}=2 \pi$ and $\mathrm{d} S=r \mathrm{~d} \theta$, we have the approximate formulas:

$$
\begin{aligned}
& \hat{f}(\boldsymbol{c}(t))=\frac{1}{N} \sum_{i=1}^{N} \hat{f}_{i}(\boldsymbol{c}(t)) \\
& \widehat{\nabla f}(\boldsymbol{c}(t))=\frac{2}{N r} \sum_{i=1}^{N} \hat{\boldsymbol{n}}_{i} \hat{f}_{i}(\boldsymbol{c}(t)) \\
& \hat{\boldsymbol{H}}_{f}(\boldsymbol{c}(t))=\frac{4}{N r^{2}} \sum_{i=1}^{N} \boldsymbol{M}_{i} \hat{f}_{i}(\boldsymbol{c}(t)),
\end{aligned}
$$

where $\hat{f}_{i}(\boldsymbol{c}(t))=f\left(\boldsymbol{c}(t)+r \hat{\boldsymbol{n}}_{i}\right)+w_{i}(t)$, with $w_{i}(t)$ the white Gaussian measurement noise affecting sensor $i$, is the $i$ th sensed value, and

$\hat{\boldsymbol{n}}_{i}=\left[\begin{array}{c}\cos i \frac{2 \pi}{N} \\ \sin i \frac{2 \pi}{N}\end{array}\right], \quad \boldsymbol{M}_{i}=\left[\begin{array}{cc}\cos i \frac{4 \pi}{N} & \sin i \frac{4 \pi}{N} \\ \sin i \frac{4 \pi}{N} & -\cos i \frac{4 \pi}{N}\end{array}\right]$.

Then, we model our vehicle with a kinematic motion of the form

$$
\left\{\begin{array}{l}
\dot{\boldsymbol{x}}(t)=v\left[\begin{array}{l}
\cos \theta(t) \\
\sin \theta(t)
\end{array}\right] \\
\ddot{\theta}(t)=u(t)
\end{array}\right.
$$


with the control reference given by

$\theta_{\text {ref }}(\boldsymbol{x}(t))=\arg \widehat{\nabla f}(\boldsymbol{c}(t))$,

where $\boldsymbol{c}$ is the center of the vehicle, that we suppose to correspond to the center of the circular sensor array. Thanks to the ease of computation of the previous formulas, we can use a control law involving the Hessian, e.g., including a damping term involving the time derivatives of both the heading angle and its reference:

$u(t)=K_{\mathrm{P}}\left[\theta_{\mathrm{ref}}(\boldsymbol{c}(t))-\theta(t)\right]+K_{\mathrm{D}}\left[\dot{\theta}_{\mathrm{ref}}(\boldsymbol{c}(t))-\dot{\theta}(t)\right]$,

where

$\dot{\theta}_{\text {ref }}(\boldsymbol{c}(t))=\frac{\widehat{\nabla f}(\boldsymbol{c}(t))^{\mathrm{T}}\left[\begin{array}{cc}0 & 1 \\ -1 & 0\end{array}\right] \hat{\boldsymbol{H}}_{f}(\boldsymbol{c}(t)) \dot{\boldsymbol{c}}(t)}{\|\widehat{\nabla f}(\boldsymbol{c}(t))\|^{2}}$

is an approximation of the angular velocity of the exact gradient.

\subsection{D vehicle dynamics and feedback design}

We consider an underactuated vehicle described by the kinematic motion law

$$
\left\{\begin{array}{l}
\dot{\boldsymbol{x}}(t)=v\left[\begin{array}{c}
\cos \theta(t) \sin \varphi(t) \\
\sin \theta(t) \sin \varphi(t) \\
\cos \varphi(t)
\end{array}\right] \\
{\left[\begin{array}{c}
\dot{\theta}(t) \\
\dot{\varphi}(t)
\end{array}\right]=\boldsymbol{u}(t),}
\end{array}\right.
$$

where $\boldsymbol{x}$ indicates the position of the center of mass of the agent, and $\theta$ and $\varphi$ are the yaw and pitch angles, respectively. In this model the forward velocity $v$ is supposed to be constant (even though other strategies can be devised), and the heading angle is controlled by the control $\boldsymbol{u}$ that steers the angular velocity. Such a choice for the vehicle's dynamics may represent, in three dimensions, a simplified model for a submarine or an autonomous underwater vehicle.

We would like to perform a gradient-ascent method using the Poisson formulas obtained in the previous sections. Our objective is to follow the gradient of the signal function $\nabla f(\boldsymbol{x}(t))$, but we consider the case where it is possible to control the angular velocity only, and not to change instantaneously the orientation of the vehicle. Thus, we define the control references as the headings given by the direction of the gradient estimated at the current point of the center of the vehicle $\boldsymbol{x}(t)$ :

$$
\left\{\begin{array}{l}
\theta_{\mathrm{ref}}(\boldsymbol{x}(t))=\arctan \frac{g_{2}(\boldsymbol{x}(t))}{g_{1}(\boldsymbol{x}(t))} \\
\varphi_{\mathrm{ref}}(\boldsymbol{x}(t))=\arccos \frac{g_{3}(\boldsymbol{x}(t))}{\|\boldsymbol{g}(\boldsymbol{x}(t))\|},
\end{array}\right.
$$

where $\boldsymbol{g}(\boldsymbol{x}(t))=\widehat{\nabla f}(\boldsymbol{x}(t))$ is the approximate gradient, so that the references are approximations of the gradient angles. Then, we define a control law with a term involving the time derivative of the reference of the heading angles:

$\boldsymbol{u}(t)=K\left[\begin{array}{c}\theta_{\mathrm{ref}}(\boldsymbol{x}(t))-\theta(t) \\ \varphi_{\mathrm{ref}}(\boldsymbol{x}(t))-\varphi(t)\end{array}\right]+\left[\begin{array}{c}\dot{\theta}_{\mathrm{ref}}(\boldsymbol{x}(t)) \\ \dot{\varphi}_{\mathrm{ref}}(\boldsymbol{x}(t))\end{array}\right]$.

The expressions for the time-derivative of the heading angles are given by

$$
\left\{\begin{array}{l}
\dot{\theta}_{\text {ref }}(\boldsymbol{x}(t))=\frac{\overline{\boldsymbol{g}}^{\mathrm{T}} \mathbf{T} \dot{\overline{\mathbf{g}}}}{\|\overline{\mathbf{g}}\|^{2}} \\
\dot{\varphi}_{\mathrm{ref}}(\boldsymbol{x}(t))=\frac{g_{3} \boldsymbol{g}^{\mathrm{T}} \dot{\boldsymbol{g}}-\dot{g}_{3}\|\boldsymbol{g}\|^{2}}{\|\boldsymbol{g}\|^{2}\|\overline{\mathbf{g}}\|},
\end{array}\right.
$$

where $\dot{\boldsymbol{g}}$ and $\boldsymbol{T}$ are defined as

$$
\dot{\boldsymbol{g}}=\frac{\mathrm{d} \widehat{\nabla f}(\boldsymbol{x}(t))}{\mathrm{d} t}=\hat{\boldsymbol{H}}_{f}(\boldsymbol{x}(t)) \dot{\boldsymbol{x}}(t), \quad \boldsymbol{T}=\left[\begin{array}{cc}
0 & 1 \\
-1 & 0
\end{array}\right]
$$

and $\overline{\mathbf{g}}:=\left[\begin{array}{lll}1 & 0 & 0 \\ 0 & 1 & 0\end{array}\right] \mathbf{g}$. We note that both $\dot{\theta}_{\text {ref }}$ and $\dot{\varphi}_{\text {ref }}$ are approximations of the respective angular velocities of the exact gradient; their expression (15) can be obtained applying the chain rule to the time derivative of (13).

In the next propositions we state the convergence of our algorithm. We consider the problem solved, and speak about convergence, if the vehicle touches the border of the source, approaches it for $t \rightarrow \infty$; in the first case, we consider our problem of interest for $t \leq t_{\mathrm{s}}$ and concluded at $t_{\mathrm{s}}$, since handling the vehicle's behavior in the neighborhood of the source, once it has been reached, is application-specific (e.g., the search vehicle might need to avoid crashing on the source).

Definition 2 (Convergence to the Source). Let the source's location be a point $\boldsymbol{x}_{\mathrm{s}} \in \Omega_{\mathrm{s}}$, with $\Omega_{\mathrm{s}}: f(\boldsymbol{x})=f_{\mathrm{s}}=\max _{\boldsymbol{x} \in \bar{\Omega}} f(\boldsymbol{x}) \forall \boldsymbol{x} \in \partial \Omega_{\mathrm{s}}$. A trajectory $\boldsymbol{x}(t)$ is said to be convergent to the source if either one of the following is true:

(I) $\exists t_{\mathrm{s}}<\infty$ such that $\boldsymbol{x}\left(t_{\mathrm{s}}\right) \in \partial \Omega_{\mathrm{s}}$;

(II) $\lim _{t \rightarrow \infty} \operatorname{dist}\left(\boldsymbol{x}(t), \partial \Omega_{\mathrm{s}}\right)=0$.

Proposition 4 (Global Convergence of the Source-Localization Algorithm). Let $\Omega=\mathbb{R}^{3} \backslash \bar{\Omega}_{\mathrm{s}}$, and let $f$ be a function harmonic in $\Omega$, continuous on $\bar{\Omega}$, and with compact sublevel sets $\Omega_{a}=\{\boldsymbol{x} \in \bar{\Omega}: f(\boldsymbol{x}) \leq$ a). Consider the system (12) under the control law (14), where the references are given by the headings of $\nabla f(\boldsymbol{x}(t))$. Then, the system converges to the source in the sense of Definition 2.

Proof. We prove that if condition I of Definition 2 is false, then condition II holds.

Let us first define the heading error as

$\boldsymbol{\gamma}(\boldsymbol{x}(t), t)=\left[\begin{array}{c}\theta_{\mathrm{ref}}(\boldsymbol{x}(t))-\theta(t) \\ \varphi_{\mathrm{ref}}(\boldsymbol{x}(t))-\varphi(t)\end{array}\right]$,

and notice that $\dot{\boldsymbol{\gamma}}(\boldsymbol{x}(t), t)=-K \boldsymbol{\gamma}(\boldsymbol{x}(t), t)$, which implies that

$\lim _{t \rightarrow \infty} \boldsymbol{\gamma}(\boldsymbol{x}(t), t)=(0,0)$

In particular, there exists a time $\bar{t}$ such that $\left|\underline{\gamma}_{1}(\boldsymbol{x}(t), t)\right| \leq \bar{\theta}$ and $\left|\gamma_{2}(\boldsymbol{x}(t), t)\right| \leq \bar{\varphi}$, with $\bar{\theta}+\bar{\varphi}<\frac{\pi}{2}$, for all $t \geq \bar{t}$.

Consider now the Lyapunov-like function

$V(\boldsymbol{x}(t))=f_{\mathrm{s}}-f(\boldsymbol{x}(t)) \geq 0$,

which is continuous on $\bar{\Omega}$, and where $V=0 \Longleftrightarrow \boldsymbol{x} \in \partial \Omega_{\mathrm{s}}$. Its time derivative along trajectories is given by

$\dot{V}(\boldsymbol{x}(t))=-\nabla f(\boldsymbol{x}(t)) \cdot \dot{\boldsymbol{x}}(t)$,

with $\dot{\boldsymbol{x}}(t)$ given by (12); after some trigonometric computation ${ }^{3}$ we can write

$\dot{V}(\boldsymbol{x}(t)) \leq-\|\nabla f(\boldsymbol{x}(t))\| v \cos (\bar{\theta}+\bar{\varphi})<0 \quad \forall t \geq \bar{t}$.

\footnotetext{
3 Given two vectors $\boldsymbol{a}_{1}, \boldsymbol{a}_{2} \in \mathbb{R}^{3}$, expressed in spherical coordinates by the pair of angles $\left(\theta_{1}, \varphi_{1}\right)$ and $\left(\theta_{2}, \varphi_{2}\right)$, such that $\left|\theta_{1}-\theta_{2}\right| \leq \bar{\theta}<\pi / 2$, and $\left|\varphi_{1}-\varphi_{2}\right| \leq$ $\bar{\varphi}<\pi / 2$, we show that $\boldsymbol{a}_{1} \cdot \boldsymbol{a}_{2} \geq a_{1} a_{2} \cos (\bar{\theta}+\bar{\varphi})$. Taking unit vectors for simplicity, computing the dot product we get $\boldsymbol{a}_{1} \cdot \boldsymbol{a}_{2}=\cos \left(\theta_{1}-\theta_{2}\right) \sin \varphi_{1} \sin \varphi_{2}+\cos \varphi_{1} \cos \varphi_{2}$. Let us start by considering the case $\cos \varphi_{1} \cos \varphi_{2} \geq 0$. Thus, $\cos \varphi_{1} \cos \varphi_{2} \geq$ $\cos \varphi_{1} \cos \varphi_{2} \cos \theta_{1}-\theta_{2}$, that implies $\boldsymbol{a}_{1} \cdot \boldsymbol{a}_{2} \geq \cos \left(\theta_{1}-\theta_{2}\right) \cos \left(\varphi_{1}-\varphi_{2}\right) \geq$ $\cos \bar{\theta} \cos \bar{\varphi} \geq \cos (\bar{\theta}+\bar{\varphi})$. Consider now the case $\cos \varphi_{1} \cos \varphi_{2}<0$. This implies $\sin \varphi_{1} \sin \varphi_{2}>0$, and hence $\sin \varphi_{1} \sin \varphi_{2} \cos \theta_{1}-\theta_{2} \geq \sin \varphi_{1} \sin \varphi_{2} \cos \bar{\theta}$; also, since $\cos \bar{\theta}+\sin \bar{\theta} \geq 1$, we have $\cos \varphi_{1} \cos \varphi_{2} \geq \cos \varphi_{1} \cos \varphi_{2} \cos \theta \sin \bar{\theta}$. Thus, $\boldsymbol{a}_{1} \cdot \boldsymbol{a}_{2} \geq \cos \bar{\theta} \cos \left(\varphi_{1}-\varphi_{2}\right)+\sin \bar{\theta} \cos \varphi_{1} \cos \varphi_{2}$. Finally, noting that $\cos \varphi_{1} \cos \varphi_{2}=$ $\cos \left(\varphi_{2}+\left(\varphi_{1}-\varphi_{2}\right)\right) \cos \varphi_{2}=\cos ^{2} \varphi_{2} \cos \left(\varphi_{1}-\varphi_{2}\right)-\cos \varphi_{2} \sin \varphi_{2} \sin \left(\varphi_{1}-\varphi_{2}\right) \geq$ $-\cos \varphi_{2} \sin \varphi_{2} \sin \left(\varphi_{1}-\varphi_{2}\right) \geq-\sin \left(\varphi_{1}-\varphi_{2}\right) \geq-\sin \bar{\varphi}$, we can write $\boldsymbol{a}_{1} \cdot \boldsymbol{a}_{2} \geq$ $\cos \bar{\theta} \cos \bar{\varphi}-\sin \bar{\theta} \sin \bar{\varphi}=\cos (\bar{\theta}+\bar{\varphi})$.
} 
If condition I is false, then $\dot{V}<0 \forall t \in[\bar{t}, \infty]$, which means that $V$ is strictly decreasing and there exists $\lim _{t \rightarrow \infty} V(\boldsymbol{x}(t))=h \geq 0$. We prove that $h=0$.

Let us suppose, by contradiction, that $h>0$. Then, $V(\boldsymbol{x}(t)) \geq$ $h \forall t \geq \bar{t}$. Since $\dot{V}$ is continuous and negative on the compact set $\{\boldsymbol{x} \in \bar{\Omega}: V(\boldsymbol{x}) \leq V(\boldsymbol{x}(\bar{t}))\} \cap\{\boldsymbol{x} \in \bar{\Omega}: V(\boldsymbol{x}(t)) \geq h\}$, it attains a maximum $-\mu$. Thus,

$$
\begin{aligned}
V(\boldsymbol{x}(t)) & =V(\boldsymbol{x}(\bar{t}))+\int_{t}^{\bar{t}} \dot{V}(\boldsymbol{x}(\tau)) \mathrm{d} \tau \\
& \leq V(\boldsymbol{x}(t))-\mu(t-\bar{t}),
\end{aligned}
$$

which implies that $\lim _{t \rightarrow \infty} V(\boldsymbol{x}(t))=-\infty$, that is a contradiction since $V(\boldsymbol{x}(t)) \geq 0 \forall t$.

Having proved that

$\lim _{t \rightarrow \infty} V(\boldsymbol{x}(t))=0$,

we want to conclude that $\operatorname{dist}(\boldsymbol{x}(t), \partial \Omega) \rightarrow 0$ as well. Let us suppose, by contradiction, that there exists $\varepsilon>0$ such that, for all $t$, there exists $T>t$ such that $\operatorname{dist}(\boldsymbol{x}(T), \partial \Omega) \geq \varepsilon$. Hence,

$$
\begin{gathered}
\boldsymbol{x}(T) \in\{\boldsymbol{x}(t): V(\boldsymbol{x}(t)) \leq V(\boldsymbol{x}(\bar{t}))\} \\
\cap\{\boldsymbol{x}(t): \operatorname{dist}(\boldsymbol{x}(t), \partial \Omega) \geq \varepsilon\},
\end{gathered}
$$

which is a compact set, so that $V$ has a minimum $m>0$ on this set. This implies that $V(\boldsymbol{x}(T)) \geq m$, thus $\exists m>0: \forall t \exists T>$ $t: V(\boldsymbol{x}(T)) \geq m$, which contradicts (18).

Remark 3. The result of Proposition 4 holds also if $\theta_{\text {ref }}$ and $\varphi_{\text {ref }}$ are perturbed estimations of the gradient angles, i.e.,

$\left\{\begin{array}{l}\theta_{\mathrm{ref}}(\boldsymbol{x}(t), t)=\theta_{\nabla f}(\boldsymbol{x}(t))+\tilde{\theta}(t) \\ \varphi_{\mathrm{ref}}(\boldsymbol{x}(t), t)=\varphi_{\nabla f}(\boldsymbol{x}(t))+\tilde{\varphi}(t),\end{array}\right.$

with $\tilde{\theta}$ and $\tilde{\varphi}$ being the uncertainties, continuous on $\bar{\Omega}$ and differentiable in $\Omega$, and such that $|\tilde{\theta}(t)|+|\tilde{\varphi}(t)| \leq \delta<\frac{\pi}{2}$. In this case, Eq. (17) in Proposition 4 becomes

$$
\begin{aligned}
\dot{V}(\boldsymbol{x}(t)) & =-\nabla f(\boldsymbol{x}(t)) \cdot \dot{\boldsymbol{x}}(t) \\
& \leq-\|\nabla f(\boldsymbol{x}(t))\| v \cos (\bar{\theta}+\bar{\varphi}+\delta) \leq 0
\end{aligned}
$$

for all $t>\bar{t}$ such that $\bar{\theta}+\bar{\varphi}<\frac{\pi}{2}$. This guarantees that $V(\boldsymbol{x}(t))$ will be decreasing, and thus that $\boldsymbol{x}(t) \rightarrow \Omega_{\mathrm{s}}$.

\subsection{Implementation details for the $3 D$ case}

The expressions for $f$, its gradient $\nabla f$ and its Hessian $\boldsymbol{H}_{f}$ in the previous formulas can be obtained from Eq. (7), considering that, using the spherical coordinates $r \hat{\boldsymbol{n}}=r(\cos \theta \sin \varphi, \sin \theta \sin \varphi$, $\cos \varphi$ ), where $\theta \in[0,2 \pi)$ and $\varphi \in[0, \pi]$ are respectively the azimuth and altitude angle, we have that $n=3, \omega_{3}=4 \pi$, and $\mathrm{d} S=r^{2} \sin \varphi \mathrm{d} \theta \mathrm{d} \varphi$, so that Eqs. (7) and (9) take the form:

$$
\begin{aligned}
f(\boldsymbol{c})= & \frac{1}{4 \pi} \int_{0}^{\pi} \sin \varphi \int_{0}^{2 \pi} f(\overline{\boldsymbol{x}}(\theta, \varphi)) \mathrm{d} \theta \mathrm{d} \varphi \\
\nabla f(\boldsymbol{c})= & \frac{3}{4 \pi r} \int_{0}^{\pi} \sin \varphi \int_{0}^{2 \pi} \hat{\boldsymbol{n}}(\theta, \varphi) f(\overline{\boldsymbol{x}}(\theta, \varphi)) \mathrm{d} \theta \mathrm{d} \varphi \\
\boldsymbol{H}_{f}(\boldsymbol{c})= & \frac{5}{4 \pi r^{2}} \int_{0}^{\pi} \sin \varphi \cdot \int_{0}^{2 \pi}\left[3 \hat{\boldsymbol{n}}(\theta, \varphi) \hat{\boldsymbol{n}}^{\mathrm{T}}(\theta, \varphi)-\boldsymbol{I}\right] \\
& \times f(\overline{\boldsymbol{x}}(\theta, \varphi)) \mathrm{d} \theta \mathrm{d} \varphi .
\end{aligned}
$$

Fig. 2(b) shows an aerial vehicle equipped with the sensing device. We can suppose that the device is solidly connected to the vehicle, and that the center of mass of the vehicle is at a small distance $\varepsilon$ from the center of the sphere, so that it will move in the direction given by $\nabla f(\boldsymbol{x}+\boldsymbol{\varepsilon}) \approx \nabla f(\boldsymbol{x})$; however, for the sake of simplicity,

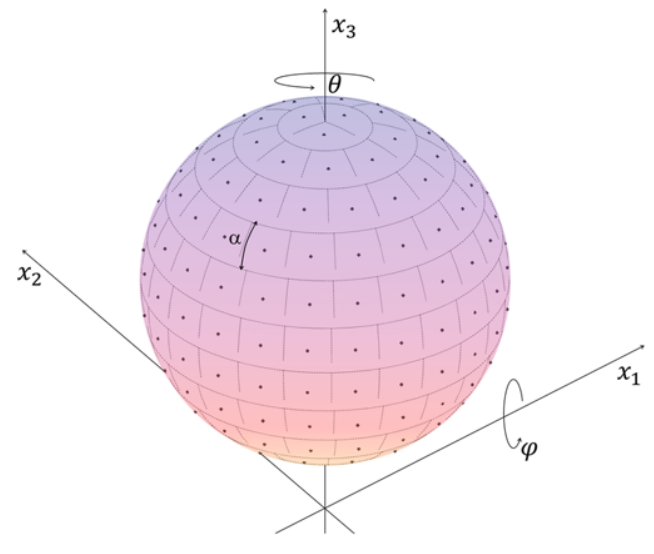

Fig. 3. Sensors placement on the spheric device $\left(\alpha=\frac{\pi}{12}\right)$.

we will assume that the two centers coincide and the gradient is estimated in the correct point.

For the computation of the integrals, we consider a suitable approximation with a discrete sum of a finite number of measurements. Differently from the two-dimensional case, where there is a very natural way to dispose the sensors (at uniformly spaced angles, see Fig. 2(a)), in three dimensions it is not trivial to choose the sensors' position. Many algorithms have been devised in the literature with the aim of finding the best partition for a spherical surface from different points of view; for a deeper study of this topic we refer to Leopardi (2006) and Saffet and Kuijlaars (1997). We choose here a simple division, that aims at maintaining a simple computation while trying to keep the sensors as equispaced as possible: given a total number of sensors $N$, we want to position them along circles ("parallels") at equispaced elevations angles $\alpha$ ("latitudes"), in a way that gives us a mesh of the sphere surface with almost regular quadrilateral elements, each having a side length $l \approx \alpha r$. This results in an angle $\bar{\alpha}=2 \sqrt{\pi / N}$, and in $N_{\mathrm{p}}=\left[\frac{\pi}{\bar{\alpha}}\right]$ parallels with latitude $\varphi_{i}=(i-0.5) \alpha, i=1, \ldots, N_{\mathrm{p}}$, where $\alpha=\pi / N_{\mathrm{p}}$ is the corrected $\bar{\alpha}$ such that the distance between two consecutive parallels is correctly estimated. Along each parallel we place a number $N_{i}$ of sensors which decreases with the distance from the equator, so as to avoid an accumulation of sensors close to the poles, since the same angular distance would result in smaller distances; thus, each sensor has an azimuthal position of $\theta_{i, j}=j \beta_{i}=j 2 \pi / N_{i}$, where $N_{i}$ is chosen such that $N_{i}=\left[\frac{2 \pi \sin \varphi_{i}}{\alpha}\right]$. As shown in Fig. 3 for $\alpha=\pi / 12$, each sensor occupies the center of an element at a position

$\overline{\boldsymbol{x}}_{i, j}:=\boldsymbol{c}+r \hat{\boldsymbol{n}}_{i, j}=\boldsymbol{c}+r\left[\begin{array}{c}\cos \theta_{i, j} \sin \varphi_{i} \\ \sin \theta_{i, j} \sin \varphi_{i} \\ \cos \varphi_{i}\end{array}\right]$.

Remark 4. We note that, using such an algorithm, $N$ cannot take all possible values, due to the approximation made to have an integer number of parallels, together with trying to have equispaced sensors; more precisely, $N$ can take values in the set $\mathcal{N}=\{6,12,20,32,46,64,82,104, \ldots\}$. This happens because our algorithm is conceived to dispose in an automatic and simple way a generic high number of sensors, but different strategies can be devised for a specific number of sensors at hand. Clearly, the sensors should be enough to guarantee a good gradient estimation. Considering the 2D scenario, a correct estimation for a field with elliptical level sets can be proven by following a similar reasoning to the Lyapunov analysis used in Moore and Canudas de Wit (2010, Theorems 1 and 2), showing that the condition $\nabla f(\boldsymbol{x}) \cdot \widehat{\nabla f} \boldsymbol{x}=$ $\frac{2}{N r} \nabla f(\boldsymbol{x}) \cdot \sum_{i=1}^{N} \hat{\boldsymbol{n}}_{i} \hat{f}_{i}(\boldsymbol{x}) \geq 0$ (corresponding to the opposite of the derivative of the Lyapunov function in the cited reference) holds for $N \geq 3$. 
For such a choice for the position of the sensors it is now possible to compute the approximate values for (19): denoting the measurement of sensor $(i, j)$ with

$\hat{f}_{i, j}(\mathbf{c}(t))=f\left(\overline{\boldsymbol{x}}_{i, j}(t)\right)+w_{i, j}(t)$,

we get

$\hat{f}(\boldsymbol{c}(t)) \approx \frac{1}{4 \pi} \sum_{i=1}^{N_{\mathrm{p}}} \alpha \sin \varphi_{i} \sum_{j=1}^{N_{i}} \beta_{i} \hat{f}_{i, j}(\boldsymbol{c}(t))$.

Then, we note that, according to the initial choice of having equilateral elements, $\beta_{i} \sin \varphi_{i}=\alpha$ (up to a small rounding error); by applying the same reasoning to (19b) and (19c), we can finally propose the following approximate formulas:

$$
\begin{aligned}
& \hat{f}(\boldsymbol{c}(t))=\frac{\alpha}{4 N_{\mathrm{p}}} \sum_{i=1}^{N_{\mathrm{p}}} \sum_{j=1}^{N_{i}} \hat{f}_{i, j}(\boldsymbol{c}(t)) \\
& \widehat{\nabla f}(\boldsymbol{c}(t))=\frac{3 \alpha}{4 N_{\mathrm{p}} r} \sum_{i=1}^{N_{\mathrm{p}}} \sum_{j=1}^{N_{i}} \hat{\boldsymbol{n}}_{i, j} \hat{f}_{i, j}(\boldsymbol{c}(t)) \\
& \hat{\boldsymbol{H}}_{f}(\boldsymbol{c}(t))=\frac{5 \alpha}{4 N_{\mathrm{p}} r^{2}} \sum_{i=1}^{N_{\mathrm{p}}} \sum_{j=1}^{N_{i}}\left(3 \hat{\boldsymbol{n}}_{i, j} \hat{\boldsymbol{n}}_{i, j}^{\mathrm{T}}-\boldsymbol{I}\right) \hat{f}_{i, j}(\boldsymbol{c}(t)) .
\end{aligned}
$$

\section{Simulations}

In this section we present some simulation results. We start by showing the result of a bi-dimensional simulation, and then study the $3 \mathrm{D}$ case more in depth, showing the behavior with the respect to the variation of some parameters, i.e., the number of sensors, the radius of ball, and noise.

\section{1. $2 D$ case}

We consider a room corresponding to problem (4). In particular, let us propose to have a rectangular room $10 \times 6 \mathrm{~m}$ wide, with a circular heater of $0.5 \mathrm{~m}$ of radius in its middle and an open window on the back-wall. The heater imposes the condition (4)(d); the walls are perfectly isolating so as to impose the Neumann boundary condition (4)(b), while the open window, $6 \mathrm{~m}$ wide, imposes the boundary condition on the value of the temperature (4)(c). Fig. 4shows the trajectories of a set of robots, described by the motion law (10), starting from different locations and with different initial orientations. The temperatures respectively of the heater and external are $T_{\mathrm{s}}=45^{\circ} \mathrm{C}$ and $T_{\text {ext }}=5{ }^{\circ} \mathrm{C}$, and each robot moves with a constant velocity of $v=0.1 \mathrm{~m} / \mathrm{s}$ and with the sensors arranged on a circumference of radius $r=0.2 \mathrm{~m}$. The control parameters in Eq. (11) are chosen as $K_{\mathrm{P}}=100$ and $K_{\mathrm{D}}=20$, and the measurements are affected by white Gaussian noise of standard deviation $\sigma=0.5$. As we can see, all vehicles reach the source, and their trajectories are perpendicular to the contour lines of the temperature; the small dithering in the robot trajectories is due to the noise in the measurements.

\section{2. $3 D$ case}

The proposed algorithm has been simulated on a scenario given by the diffusion of an isotropic source emitting at a constant rate in an unbounded domain (unperturbed by borders), a simplified model of a pollutant leakage underwater in a wide sea or ocean, in which a sensing device is seeking a source whose signal decays away from the source according to the expression (3), where $\boldsymbol{x}_{\mathrm{s}}$ is the position of the source, and $A$ is a coefficient whose choice influences the amplitude of the signal. The device, which has no

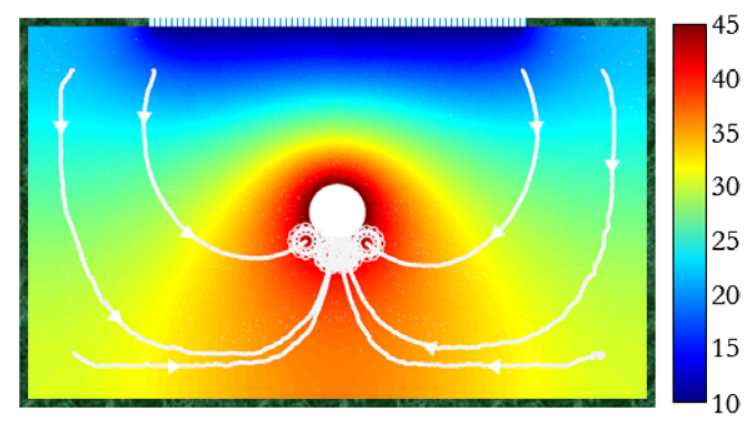

Fig. 4. Result of a simulation for the bidimensional scenario. The colors' shades represent the temperature profile $\left({ }^{\circ} \mathrm{C}\right)$; the white lines are the trajectories of the vehicles. (For interpretation of the references to color in this figure legend, the reader is referred to the web version of this article.)

Table 1

Mean values of the parameters (21) for simulations for a varying $N$.

\begin{tabular}{rlll}
\hline$N$ & $\mathrm{E}[\tilde{v}](\mathrm{m} / \mathrm{s})$ & $\mathrm{E}\left[J_{\theta}\right]\left(^{\circ}\right)$ & $\mathrm{E}\left[J_{\varphi}\right]\left(^{\circ}\right)$ \\
\hline 6 & - & - & - \\
12 & 0.0290 & 0.1479 & 3.0034 \\
20 & 0.0845 & 0.0700 & 2.6763 \\
32 & 0.0977 & 0.0279 & 2.6372 \\
46 & 0.0990 & 0.0202 & 2.6018 \\
64 & 0.0992 & 0.0077 & 2.6096 \\
82 & 0.0993 & 0.0049 & 2.5895 \\
\hline
\end{tabular}

knowledge about $f(\boldsymbol{x})$, has a constant velocity $v=.1 \mathrm{~m} / \mathrm{s}$, and the control parameter is set as $K=0.95$. The initial orientations of the vehicle are chosen as $\theta(0)=\arctan \left(c_{2}(0) / c_{1}(0)\right)$ and $\varphi(0)=$ $\arccos \left(c_{3}(0) /\|\boldsymbol{c}(0)\|\right)$, and the initial angular velocities are set as $\dot{\theta}(0)=\dot{\varphi}(0)=0$. The amplitude of the signal function is chosen to be $A=25$

\subsubsection{Sensitivity to $N$ and $r$}

To study the impact of the number of sensors on the convergence of the vehicle to the source, we simulated the same scenario for up to 100 sensors, i.e., for $N \in\{6,12,20,32,46,64,82\}$. Every vehicle, having a unit radius, starts from the same initial position, and the source is set at the origin. We run a Monte Carlo simulation with 100 different initial positions for each value of $N$, and compute the mean of the average velocity and of the errors on the yaw and pitch angles, defined as

$$
\begin{aligned}
\tilde{v}(N) & =\frac{\|\boldsymbol{c}(0)\|-r}{T} \\
J_{\theta}(N) & =\frac{1}{T} \sum_{t=1}^{T}|\theta(t)-\hat{\theta}(t)| \\
J_{\varphi}(N) & =\frac{1}{T} \sum_{t=1}^{T}|\varphi(t)-\hat{\varphi}(t)| .
\end{aligned}
$$

The results are summarized in Table 1 . For $N=6$ there is no convergence, while for the other configurations, the largest error on the estimated direction is of about $3^{\circ}$, even for $N=12$. Fig. 5(a) shows an example of the trajectories, for the same starting point and three different values of $N$.

Remark 5. We note that with 6 sensors the algorithm fails because of the specific way in which they are automatically placed (see Remark 4). An ad hoc configuration for $N=6$ that provides convergence is given by placing two sensors in correspondence of the elevation angles $\varphi=0$ and $\varphi=1$, and four of them equispaced on the great circle of elevation $\varphi=\pi / 2$. 


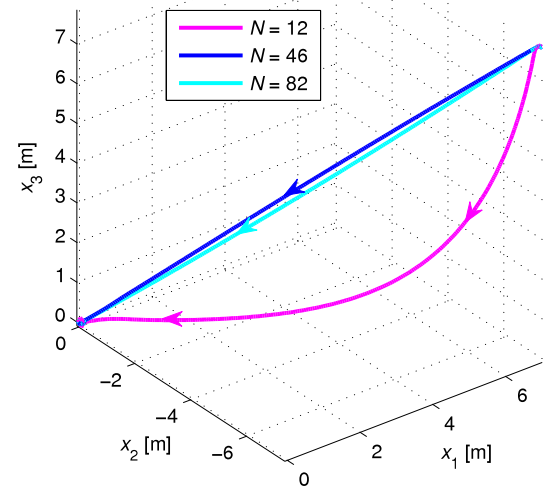

(a) Varying $N$.

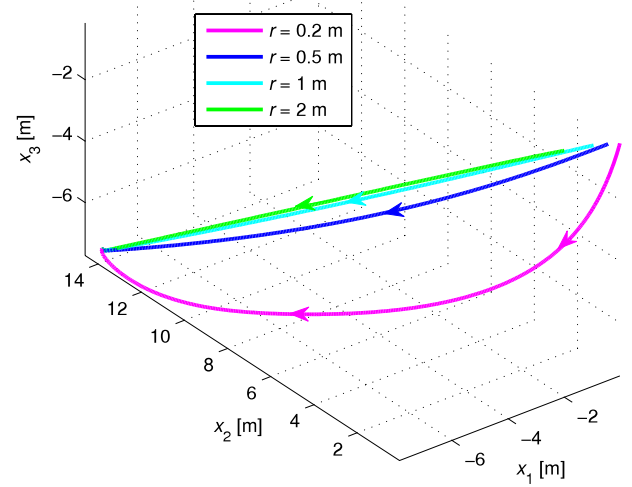

(b) Varying $r$.

Fig. 5. Trajectories of a source-seeking vehicle for different numbers of sensors and radius length.

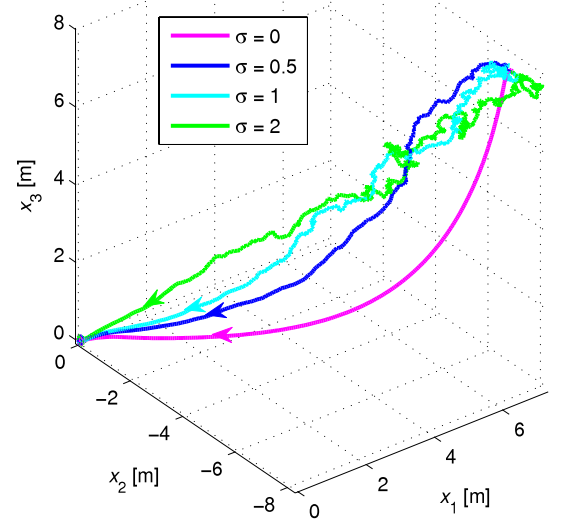

(a) $N=12$, varying $\sigma$.

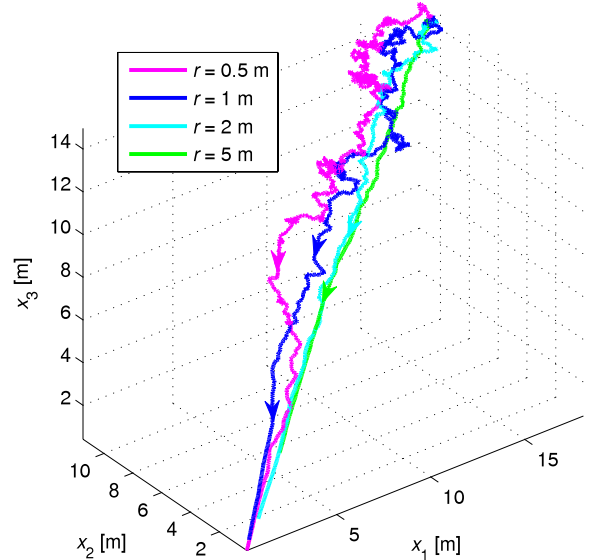

(b) $N=46, \sigma=0.75$, varying $r$.

Fig. 6. Trajectories of a source-seeking vehicle with measurements corrupted by noise.

Fig. 5(b) depicts the trajectories for a varying length of the radius $r$ of the ball on which the sensors are placed, and a fixed value of $N=46$; we kept the same values as before for the other parameters. As we can see, the trajectory is better when the radius is not too small. Indeed, while formula (19b) is exact regardless of the radius value, when we approximate it with a finite sum a bigger radius is useful to attenuate the effect of quantization (and possibly of measurement noise).

\subsubsection{Noise influence}

We consider now the case where each sensor gives a measurement of the emitted signal corrupted by noise. We fix the values of $N$ and $r$ to $N=46$ and $r=1 \mathrm{~m}$, and run a Monte Carlo simulation with sensors affected by a white Gaussian noise of increasing standard deviation $\sigma$. For each value of $\sigma$, we run 100 simulations; the simulation was considered to be failed if time got over 10 times the minimum time to reach the source $t^{*}=\|\boldsymbol{c}(0)\| / v$, and the process stopped when all the trials, for a given $\sigma$, failed. The result is summarized in Table 2, while in Fig. 6(a) we can see the trajectories resulting from a simulation with $N=12$, and some different values of $\sigma$. We can see that, except for the oscillation due to the noise, the vehicles settle on a small ball around the source.

The results of a simulation for $N=46, \sigma=0.75$ and different values of $r$ are reported in Fig. 6(b).

\subsubsection{Nonharmonic diffusion process}

The proposed method relies on the assumptions of an isotropic source, and of the harmonicity of the solution of the PDE associated to the diffusion process. Experimental results suggest that the method may have a good behavior also when the properties are not fulfilled, if the gradient error introduced by the nonharmonicity is bounded, as shown in Proposition 4. Another class of functions for which the gradient estimated with the Poisson formula is exact, although such functions are in general nonharmonic, is represented by quadratic functions.

We propose here a simulation example when these assumptions are not satisfied. The vehicle is equipped with 20 sensors disposed on a sphere with unit radius, and is seeking a source whose signal decays according to the non-harmonic function

$\bar{f}(\boldsymbol{x})=25 \mathrm{e}^{-\frac{2.2 x_{1}^{2}+0.7 x_{2}^{2}+1.3 x_{3}^{2}}{10}}$.

Fig. 7 depicts the result of such simulation, for the same control constants as before. As a more realistic example we refer also to a practical implementation of a bidimensional source-seeking robot, whose videos are available at the NeCS website. ${ }^{4}$ In this setting the sensing device is given by a camera, and the robot is moving on a

4 http://necs.inrialpes.fr/pages/platforms.php\#sourceLoc 
Table 2

Percentage of successful simulations, and mean of the normalized time to reach the source, for increasing noise.

\begin{tabular}{|c|c|c|c|c|c|c|c|c|c|c|c|c|}
\hline$\sigma$ & 0.5 & 1 & 1.5 & 2 & 2.5 & 3 & 3.5 & 4 & 4.5 & 5 & 5.5 & 6 \\
\hline$\%$ & 100 & 100 & 100 & 91 & 73 & 47 & 35 & 21 & 9 & 10 & 7 & 4 \\
\hline $\mathrm{E}\left[\frac{t}{t^{*}}\right]$ & 2.0469 & 3.6287 & 5.3099 & 6.3628 & 7.2335 & 7.7745 & 8.1791 & 8.5948 & 8.2819 & 8.5013 & 8.7760 & 9.1355 \\
\hline
\end{tabular}
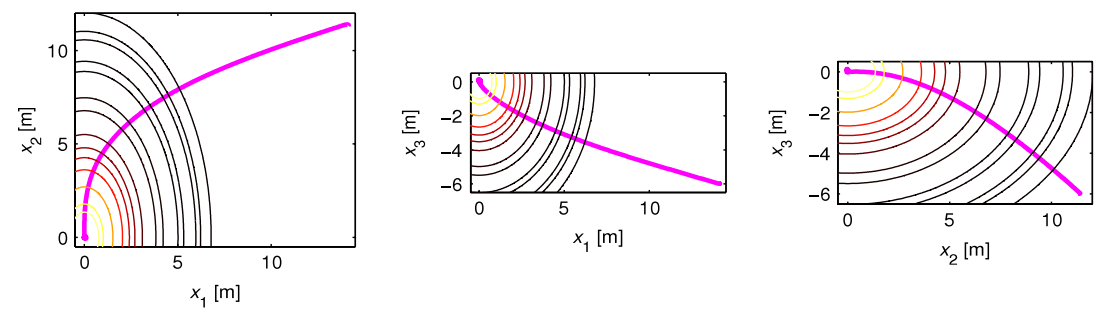

Fig. 7. Trajectory for a vehicle seeking a source whose diffusion is described by the nonharmonic function $\bar{f}(\boldsymbol{x})$.

thermal representation of a discharge of industrial water, whose dispersion is nonharmonic.

\section{Conclusions}

We have proposed a method for solving the source-seeking task by a gradient estimation based on the Poisson integral. This technique allows for computing derivatives of any order via simple integrals, and the approach demonstrated to be easily adaptable to different dimensions (i.e., for planar or spatial seeking tasks). Although the three-dimensional implementation presents additional problems to be faced, such as the sensors' placement, the generic mathematical framework can be extended without any substantial change. The method, which showed to be robust to noise, is developed under the main assumption of the harmonicity of the solution of the PDE describing the diffusion process; nevertheless, some simulations showed convergence to the source even in cases in which this assumption was not satisfied.

\section{Acknowledgments}

The authors would like to thank Pierre Rouchon, for enriching discussions giving rise to this work, and Antoine Rousseau, for the FreeFem ++ code used for the finite elements simulation.

\section{References}

Axler, S., Bourdon, P., \& Ramey, W. (2001). Harmonic function theory. New York, USA: Springer-Verlag.

Baronov, D., \& Baillieul, J. (2008). Autonomous vehicle control for ascending/descending along a potential field with two applications. In Proceedings of the 2008 American control conference (pp. 678-683). Seattle, USA.

Briñón Arranz, L., Seuret, A., \& Canudas de Wit, C. (2011). Collaborative estimation of gradient direction by a formation of AUVs under communication constraints. In Proceedings of the 50th IEEE conference on decision and control and European control conference (pp. 5583-5588). Orlando, USA. December.

Burian, E., Yoerger, D., Bradley, A., \& Singh, H. (1996). Gradient search with autonomous underwater vehicles using scalar measurements. In Proceedings of the 1996 symposium on autonomous underwater vehicle technology (pp. 86-98). Monterey, USA.

Canudas de Wit, C., Garin, F., Fabbiano, R., Rouchon, P., \& Rousseau, A. (2012). Source localization using Poisson integrals. In Proceedings of the 3rd IFAC workshop on distributed estimation and control in networked systems. Santa Barbara, USA. September.

Cochran, J., Ghods, N., \& Krstić, M. (2008). 3D nonholonomic source seeking without position measurement. In Proceedings of the 2008 American control conference (pp. 3518-3523). Seattle, USA. June.

Cochran, J., Siranosian, A. A., Ghods, N., \& Krstić, M. (2009). 3-D source seeking for underactuated vehicles without position measurement. IEEE Transactions on Robotics, 25(1), 117-129.

Folland, G. B. (1995). Introduction to partial differential equations. Princeton Academic Press.
Ghods, N. (2011). Extremum-seeking for mobile robots. (Ph.D. thesis). San Diego: University of California.

Ghods, N., \& Krstić, M. (2010). Multi-agent deployment arounde a source in one dimension by extremum seeking. In Proceedings of the 2010 American control conference (pp. 4794-4799). Baltimore, USA.

Khapalov, A. (2010). Source localization and sensor placement in environmental monitoring. International Journal of Applied Mathematics and Computer Science, 20(3), 445-458.

Leopardi, P. (2006). A partition of the unit sphere into regions of equal area and small diameter. Electronic Transactions on Numerical Analysis, 25, 309-327.

Matthes, J., Gröll, L., \& Keller, H. B. (2004). Source localization based on pointwise concentration measurements. Sensors and Actuators A: Physical, 115, 32-37.

Menon, P.P., \& Ghose, D. (2012). Simultaneous source localization and boundary mapping for contaminants. In Proceedings of the 2012 American control conference (pp. 4174-4179). Montréal, Canada. June.

Mesquita, A. R., Hespanha, J., \& Åström, K. (2008). Optimotaxis: a stochastic multiagent optimization procedure with point measurements. In Proceedings of the 11th international workshop on hybrid systems: computation and control. Saint Louis, USA: Springer.

Moore, B.J., \& Canudas de Wit, C. (2010). Source-seeking via collaborative measurements by a circular formation of agents. In Proceedings of the 2010 American control conference (pp. 6417-6422). Baltimore, USA.

Moreau, L., Bachmayer, R., \& Leonard, N.E. (2003). Coordinated gradient descent: a case study of Lagrangian dynamics with projected gradient information. In Proceedings of the 2nd IFAC workshop on Lagrangian and Hamiltonian methods in nonlinear control (pp. 57-62). Sevilla, Spain. April.

Porat, B., \& Nehorai, A. (1996). Localizing vapor-emitting sources by moving sensors. IEEE Transactions on Signal Processing, 44(4), 1018-1021.

Rabbat, M., \& Nowak, R. (2004). Distributed optimization in sensor networks. In Proceedings of the 3rd international symposium on information processing on sensor networks (pp. 20-27). Berkeley, USA.

Saffet, E. B., \& Kuijlaars, A. B. J. (1997). Distributing many points on a sphere. The Mathematical Intelligencer, 19(1), 5-11.

Sahyoun, S.S., Djouadi, S.M., \& Qi, H. (2010). Dynamic plume tracking using mobile sensors. In Proceedings of the 2010 American control conference (pp. 2915-2920). Baltimore, USA.

Wu, W., Chang, D., \& Zhang, F. (2013). A bio-inspired robust 3D plume-tracking strategy using mobile sensor networks. In Proceedings of the 52nd IEEE conference on decision and control. Florence, Italy. December.

Wu, W., Couzin, I.D., \& Zhang, F. (2012). Bio-inspired source-seeking with no explicit gradient estimation. In Proceedings of the 3rd IFAC workshop on distributed estimation and control in networked systems. Santa Barbara, USA. September.

Yong, Z., Qing-Hao, M., Yuxiu, W., \& Ming, Z. (2012). Distributed gas-source localization based on MMSE estimation with cooperative sensor networks. In Proceedings of the 31st Chinese control conference (pp. 6611-6616). Hefei, China. July.

Zhang, C., Florêncio, D., Ba, D. E., \& Zhang, Z. (2008). Maximum likelihood sound source localization and beamforming for directional microphone arrays in distributed meetings. IEEE Transactions on Multimedia, 10(3), 538-548.

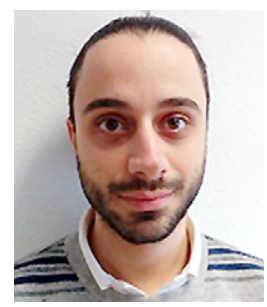

Ruggero Fabbiano is currently a Ph.D. candidate in Automatic Control with the NeCS team at Inria RhôneAlpes, Montbonnot Saint Martin (France). He was born in Bergamo (Italy), and received his B.S. and M.S. degrees in Computer Engineering from Università degli Studi di Pavia (Italy), in 2008 and 2011 respectively. His research interests are in source localization and distributed control. 


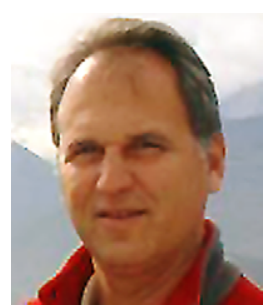

Carlos Canudas de Wit was born in Villahermosa, Tabasco, Mexico in 1958. He received his B.Sc. degree in Electronics and Communications from the Technological Institute of Monterrey, Mexico in 1980. In 1984 he received his M.Sc. from the Department of Automatic Control of the University of Grenoble, France. He was a visiting researcher in 1985 at Lund Institute of Technology, Sweden. In 1987 he received his Ph.D. in Automatic Control from the Polytechnic of Grenoble, France. Since then he has been working at the same department as "Directeur de recherche" at the CNRS, where he teaches and conducts research in the area of nonlinear control of mechanical systems and networked controlled systems. He is the current leader of the GIPSA-lab/Inria NeCS team on networked controlled systems. He has been an associate editor of the IEEE Transactions on Automatic Control, from Jan. 1992 to Dec. 1997, and of Automatica, from 1999 to 2002, and editor of the Asian Journal of Control (since 2010), the IEEE Transactions on Control System Technology (since Jan. 2013) and of the IEEE
Transactions on Control of System Networks (since June 2013). He is the current president of the EUCA association for the period 2013-'15, and serves at the IEEE Board of Governors of the Control System Society 2011-2014. His research publications include 185 international conference papers and 62 published papers in international journals, 5 books, 9 book chapters, and 11 patents.

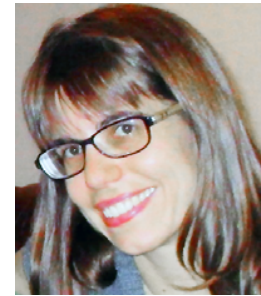

Federica Garin is "chargée de recherche" (researcher) with the NeCS team at Inria Rhône-Alpes, Grenoble (France). She received her B.Sc., M.S., and Ph.D. degrees in Applied Mathematics from Politecnico di Torino (Italy) in 2002, 2004, and 2008, respectively. She was a postdoctoral researcher at DEI, Università di Padova (Italy) in 2008 and 2009, and at Inria Rhône-Alpes in 2010. Her current research interests are in distributed algorithms for sensor networks and networked control. 